\title{
Cyclic Sieving, Promotion, and Representation Theory
}

\author{
Brendon Rhoades
}

Department of Mathematics, University of Minnesota, Minneapolis, MN, 55455

\begin{abstract}
We prove a collection of conjectures due to Abuzzahab-Korson-Li-Meyer, Reiner, and White regarding the cyclic sieving phenomenon as it applies to jeu-de-taquin promotion on rectangular tableaux. To do this, we use Kazhdan-Lusztig theory and a characterization of the dual canonical basis of $\mathbb{C}\left[x_{11}, \ldots, x_{n n}\right]$ due to Skandera. Afterwards, we extend our results to analyzing the fixed points of a dihedral action on rectangular tableaux generated by promotion and evacuation, suggesting a possible sieving phenomenon for dihedral groups. Finally, we give applications of this theory to cyclic sieving phenomena involving reduced words for the long elements of hyperoctohedral groups, handshake patterns, and noncrossing partitions.
\end{abstract}

Keywords: Kazhdan-Lusztig theory, combinatorial actions, tableaux

\section{Introduction}

Let $X$ be a finite set, $C=\langle c\rangle$ be a cyclic group acting on $X$, and $X(q)$ be a polynomial in $\mathbb{Z}[q]$. Let $\zeta \in \mathbb{C}$ be a primitive $|C|^{t h}$ root of unity. Following Reiner, Stanton, and White (15), we say that the triple $(X, C, X(q))$ exhibits the cyclic sieving phenomenon (CSP) if for all $d \in \mathbb{N},\left|X^{c^{d}}\right|=X\left(\zeta^{d}\right)$. That is, the cardinality of the fixed set $X^{c^{d}}$ is given by the polynomial $X(q)$ evaluated at $q=\zeta^{d}$. In particular, $X(1)=|X|$.

In their paper, Reiner et. al. give several examples of cyclic actions and polynomials which exhibit the CSP. For example, if we let $X$ be the set of $k$-subsets of $[n]$ and $C=\mathbb{Z} / n \mathbb{Z}$ act on $X$ by the long cycle $(1,2, \ldots, n) \in S_{n}$, then the triple $\left(X, C,\left[\begin{array}{l}n \\ k\end{array}\right]_{q}\right)$ exhibits the CSP, where $\left[\begin{array}{l}n \\ k\end{array}\right]_{q}$ is the $q$-analogue of the binomial coefficient $\left(\begin{array}{l}n \\ k\end{array}\right)$. That is, $\left[\begin{array}{c}n \\ k\end{array}\right]_{q}=\frac{[n !]_{q}}{[k !]_{q}[(n-k) !]_{q}}$, where $[m !]_{q}:=[m]_{q}[m-1]_{q} \cdots[1]_{q}$ and $[j]_{q}:=\frac{1-q^{j}}{1-q}$. The same authors exhibit another CSP by letting $X$ be the set of all triangulations of the $n$-gon and $C=\mathbb{Z} / n \mathbb{Z}$ act on $X$ by rotation. Again in this case $X(q)$ is equal to the $q$-analogue of $|X|$.

Given a partition $\lambda \vdash n$, let $S Y T(\lambda)$ denote the set of all standard Young tableaux of shape $\lambda$. It is known that jeu-de-taquin promotion defines a cyclic action on the set $S Y T(\lambda)$. Moreover, the hook length 1365-8050 @ 2008 Discrete Mathematics and Theoretical Computer Science (DMTCS), Nancy, France 
formula is a well-known way to compute $f^{\lambda}:=|S Y T(\lambda)|$ given by

$$
f^{\lambda}=\frac{n !}{\Pi_{(i, j) \in \lambda} h_{i j}},
$$

where $h_{i j}$ is the hook length in $\lambda$ corresponding to the position $(i, j)$. In the pattern of the above examples, one might suspect that there is another instance of a CSP with $X=S Y T(\lambda)$, the cyclic group being the action of jeu-de-taquin, and $X(q)$ the $q$-analogue of the hook length formula,

$$
X(q)=\frac{[n !]_{q}}{\Pi_{(i, j) \in \lambda}\left[h_{i j}\right]_{q}} .
$$

Unfortunately, this conjecture is false in general. It is possible to show that it holds for hook shaped $\lambda$, and in this case reduces to the aforementioned CSP for $k$-subsets of $[n]$. However, even for the shape $\lambda=(3,2)$ the aforementioned conjecture does not hold. The first main theorem of this paper proves a conjecture of Reiner et. al. this this holds when $\lambda$ is a rectangle. We also prove a related conjecture of the same authors which predicted a CSP given by letting $X:=C S T(\lambda, k)$, the set of column strict tableaux of a rectangular shape $\lambda$ with entries bound by some number $k \geq \ell\left(\lambda^{\prime}\right)$, letting jeu-de-taquin promotion act on $X$, and letting $X(q)$ be (up to a power of $q$ ) the principal specialization of the Schur function $s_{\lambda}$ given by $s_{\lambda}\left(1, q, \ldots, q^{k-1}\right)$. (It should be noted that, for us, the entries in a column strict tableaux will increase weakly across rows and strictly down columns. A tableau $T$ is row strict if and only if the conjugate $T^{\prime}$ is column strict and the set of row strict tableaux of shape $\lambda$ and entries bounded by $k$ is $\operatorname{RST}(\lambda, k)$.)

While CSPs are combinatorially interesting phenomena in and of themselves, they often allude to deeper algebraic structure. Often, while the initial proof of a CSP is a brute force counting argument, further analysis reveals that a more elegant and revealing algebraic approach exists. One tool used in the proof of the CSP on $\left(\begin{array}{c}{[n]} \\ k\end{array}\right)$ was Springer's results (21) on regular elements, and this shall be used again in our work. Our main strategy throughout will be to model the action of promotion via representations.

More specifically, let $X$ be either the set $S Y T(\lambda)$ or the $C S T(\lambda, k)$. In either case, we shall form a representation of degree $|X|$ with basis vectors $f_{x}$ indexed by elements $x \in X$. We will also give an operator $T$ in the image of this representation such that, up to a predictable sign, we have that $T\left(f_{x}\right)=$ $f_{j(x)}$ always, where $j(x)$ is the image of $x$ under jeu-de-taquin promotion. With this set-up, counting the number of fixed points of various powers of the operator $j$ is equivalent to evaluating the trace of various powers of $T$ and this latter problem can be attacked with the techniques of representation theory.

A hint at how to do this for the case $X=S Y T(\lambda)$ is provided by the work of Berenstein and Zelevinsky (3) and Stembridge (24) regarding Stembridge's $q=-1$ phenomenon. The $q=-1$ phenomenon is the special case of a CSP where the cyclic group has order 2. In this case, the specification of the cyclic action is equivalent to specifying an involution on the set which is acted upon. Evacuation on either standard or column strict tableaux was one such involution considered by Stembridge. Given $\lambda \vdash n$ an arbitrary partition, we have the Kazhdan-Lusztig (left) cellular representation corresponding to $\lambda$ which has basis vectors identified with $S Y T(\lambda)$ and is isomorphic as an $S_{n}$-module to the Specht module $S^{\lambda}$ corresponding to $\lambda$. 
Theorem 1.1 (Berenstein, Zelevinsky (3), Stembridge (24)) Denote the $\lambda$-KL cellular representation by $\rho$. Let $w_{o} \in S_{n}$ be the long element.

Then, up to a plus or minus sign, we have that $\rho\left(w_{o}\right)$ is the linear operator which sends $P$ to $e(P)$, where e is evacuation.

We prove an analogous result (Proposition 3.5) which requires $\lambda$ to be rectangular, replaces $e$ with $j$, and replaces the long element $w_{o} \in S_{n}$ with the long cycle $c_{n}=(1,2, \ldots, n) \in S_{n}$. The rectangularity of $\lambda$ is essential in the proof of Proposition 3.5 for reasons related to a symmetry of the KL $\mu$-function (Proposition 3.2), a cyclic action on a new combinatorial object called the 'extended descent set' related to rectangular tableaux (Lemma 3.3), and the preservation of irreducibility of $S_{n}$-modules corresponding to rectangular shapes upon restriction to $S_{n-1}$.

The proof of the column strict conjecture builds from the case of standard tableaux. We first consider a basis of an $S_{n}$-module dual to the KL cellular basis which can be interpreted in terms of images of certain polynomials called 'immanants' under a certain projection map. This leads to a basis indexed by column strict tableaux for a module over the general linear group which turns out to be irreducible of highest weight $\lambda$. This uses a characterization of Lusztig's dual canonical basis due to Skandera (20).

There exist fancier versions of the CSP in which the group acting is not cyclic. Barcelo, Reiner, and Stanton (2) consider an action of a product of two cyclic groups on complex reflection groups and prove a 'bicyclic sieving phenomenon'. In our situation, it follows that for rectangular shapes $\lambda$, we have a dihedral action on any of $S Y T(\lambda)$, column strict tableaux with uniformly bounded entries, or row strict tableaux with uniformly bounded entries given by the group generated by the actions of $j$ and $e$. We determine the size of the fixed point set corresponding to any conjugacy class of this dihedral group, interpreting this as a step towards an occurrence of a 'dihedral sieving phenomenon'. This result proves a conjecture of Abuzzahab, Korson, $\mathrm{Li}$, and Meyer (1) and re-proves one of their theorems, as well as a theorem of Stembridge (24).

The organization of this paper is as follows. In Section 2 we review some background regarding compositions, partitions, tableaux, and Kazhdan-Lusztig theory. In Section 3 we prove our CSP for the case of standard tableaux. In Section 4 we prove our CSPs for column strict tableaux. In Section 5 we prove results concerning the dihedral action of promotion and evacuation. In Section 6 we apply our results to obtain a CSP for a cyclic action on the set of reduced words for the long element of the Coxeter group $B_{n}$ of signed permutations of $[n]$. In Section 6 we also use our results to get a CSP for noncrossing partitions and handshake patterns. Most proofs are omitted for this extended abstract; for more details, see (17).

\section{Background}

A more detailed exposition of the material in this section can be found in, for example, (22), (23), (19), and (9). Given $n \in \mathbb{N}$, by a composition $\alpha$ of $n$ we mean a finite sequence of nonnegative integers $\left(\alpha_{1}, \ldots, \alpha_{k}\right)$ such that $\alpha_{1}+\cdots+\alpha_{k}=n$ (some of the $\alpha_{i}$ are allowed to be zero). Define the length of $\alpha$ to be $\ell(\alpha):=k$. Write $\alpha \models n$ to denote that $\alpha$ is a composition of $n$. Also write $c_{k} . \alpha$ for the composition $\left(\alpha_{k}, \alpha_{1}, \ldots, \alpha_{k-1}\right)$ of $n$ and $w_{o} \dot{\alpha}$ for the composition $\left(\alpha_{k}, \alpha_{k-1}, \ldots, \alpha_{1}\right)$ of $n$. Any composition $\alpha \models n$ of length $k$ defines a function $[n] \rightarrow[k]$, also denoted by $\alpha$, given by $\alpha(i)=j$ if $i \in\left(\alpha_{1}+\cdots+\alpha_{i-1}, \alpha_{1}+\cdots+\alpha_{i}\right]$. For example, if $n=6$ and $k=7$ and $\mu$ is the composition of 6 
given by $(0,2,0,0,1,3,0)$, we have that the associated function $[6] \rightarrow[7]$ is given by $\alpha(1)=\alpha(2)=2$, $\alpha(3)=5$, and $\alpha(4)=\alpha(5)=\alpha(6)=6$. A partition of $n$ is a weakly decreasing composition of $n$ with no entries equal to zero. Define a statistic $\kappa$ on partitions by $\kappa(\lambda)=0 \lambda_{1}+1 \lambda_{2}+2 \lambda_{3}+\cdots$, where $\lambda=\left(\lambda_{1}, \ldots, \lambda_{k}\right) \vdash n$.

For any composition $\alpha \models n$ with $\ell(\alpha)=k$, define $C S T(\lambda, k, \alpha)$ (resp. $R S T(\lambda, k, \alpha)$ ) to be the set of column strict (resp. row strict) tableaux of shape $\lambda$ and content $\alpha$. If $\lambda \vdash n$ is a non-skew shape and $T \in S Y T(\lambda)$, write $D(T)$ for the descent set of $T$.

For each $i \in[n-1]$ we denote by $s_{i}$ the adjacent transposition $(i, i+1)$ in the symmetric group $S_{n}$. In this paper, writing $u \leq w$ for permutations $u$ and $w$ will always mean that $u$ and $w$ are comparable in Bruhat order. Given permutations $u$ and $w$, define $\ell(u, w)=\ell(w)-\ell(u)$ to be the difference in lengths between $u$ and $w$. We will sometimes identify permutations with their one-line notation $w=w_{1} w_{2} \ldots w_{n}$, meaning that $w$ sends 1 to $w_{1}, 2$ to $w_{2}$, and so on. We let $s_{i}$ act on $S_{n}$ on the left by swapping the letters in positions $i$ and $i+1$, whatever those letters may be. For example, $s_{2} \cdot 142563=124563$. Define the long element $w_{o} \in S_{n}$ to be the permutation whose one-line notation is $n(n-1) \ldots 1$. For two standard tableaux $P$ and $Q$ of shape $\lambda$, we will always write $w \mapsto(P, Q)$ to denote that the permutation $w$ maps to $(P, Q)$ under RSK row insertion.

Given a tableau $T \in C S T(\lambda, k, \alpha)$, define the image $j(T)$ of $T$ under jeu-de-taquin promotion $j$ to be the element of $C S T\left(\lambda, k, c_{k} . \alpha\right)$ obtained by replacing every $k$ in $T$ by a dot, playing jeu-de-taquin on $T$ to move all of the dots into the upper left hand corner, increasing every entry of the resulting tableau by 1 , and replacing every dot with a 1 . Observe that $j(T)$ is in particular well defined for $T \in S Y T(\lambda)(=$ $\left.C S T\left(\lambda, n, 1^{n}\right)\right)$ and maps $S Y T(\lambda)$ to itself. If $T \in R S T(\lambda, k, \alpha)$, we have that $T^{\prime} \in C S T\left(\lambda^{\prime}, k, \alpha\right)$ and $j(T):=j\left(T^{\prime}\right)^{\prime}$. Also denote by $e$ the operation of Schützenberger evacuation on either column strict or standard tableaux.

Kazhdan and Lusztig studied representations of Hecke algebras in (12) and introduced a basis for the Hecke algebra $H_{n}(q)$ which specializes at $q=1$ to a basis for the classical group algebra $\mathbb{C}\left[S_{n}\right]$. The definitions of these bases involve certain polynomials $P_{u, v}(q) \in \mathbb{N}[q]$ defined for ordered pairs of permutations $(u, v)$ called the $K L$ polynomials. These polynomials have been studied extensively ((5), (7), (8)) and we record a couple of their basic properties here.

Lemma 2.1 Let $u, w$ be permutations in $S_{n}$. We have that $P_{u, w}(q) \neq 0$ if and only if $u \leq w$ and the degree of $P_{u, w}(q)$ is at most $\frac{\ell(w)-\ell(u)-1}{2}$.

In light of the above result, define $\mu(u, w)$ to be the coefficient of $q^{\frac{\ell(w)-\ell(u)-1}{2}}$ in $P_{u, w}(q)$. That is, $\mu(u, w)$ is the coefficient of the maximum possible power of $q$ in $P_{u, w}(q)$, if it appears. We shall also use a symmetrized version $\mu[u, w]$ of $\mu$ defined by $\mu[u, v]:=\max (\mu(u, v), \mu(v, u))$.

We have the following change-of-label result which relates the $\mu$ function to the RSK algorithm. (9)

Lemma 2.2 Let $\lambda \vdash n$ and let $P, Q, S$, and $T$ be standard tableaux of shape $\lambda$. Identify permutations with their images under $R S K$. 
We have that

$$
\begin{aligned}
& \mu[(P, S),(Q, S)]=\mu[(P, T),(Q, T)], \\
& \mu[(P, S),(P, T)]=\mu[(Q, S),(Q, T)] .
\end{aligned}
$$

In light of the above lemma, we define $\mu[P, Q]$ for two standard tableaux $P$ and $Q$ of the same shape to be the number $\mu[(P, T),(Q, T)]$, where we identify permutations with their images under RSK and $T$ is an arbitrary standard tableau of the same shape as $P$ and $Q$.

Kazhdan-Lusztig theory leads to an important avatar of the irreducible representations of the symmetric group. Fix a partition $\lambda \vdash n$ and define an action of the adjacent transposition $s_{i}$ on the vector space $\mathbb{C}[S Y T(\lambda)]$ by:

$$
s_{i} P= \begin{cases}-P & \text { if } i \in D(P) \\ P+\sum_{i \in D(Q)} \mu[P, Q] Q & \text { if } i \notin D(P)\end{cases}
$$

for $i \in[n-1]$ and $P \in S Y T(\lambda)$.

Remarkably, this action of the adjacent transpositions $s_{i}$ respects the Coxeter relations for the symmetric group $S_{n}$ and so makes $\mathbb{C}[S Y T(\lambda)]$ into an $S_{n}$-module. Again remarkably, this module is isomorphic to the irreducible representation of $S_{n}$ indexed by $\lambda$ and called the (left cellular) KL representation corresponding to $\lambda$.

\section{Promotion on Standard Tableaux}

Given a rectangular shape $\lambda=b^{a}$ with $a b=n$, we want to determine how the operation of jeu-de-taquin promotion on $S Y T(\lambda)$ interacts with the left KL cellular representation $S^{\lambda}$ of shape $\lambda$. Our first goal is to show that the promotion operator $j$ interacts nicely with the $\mu$ function. Define a deletion operator $d: S Y T\left(b^{a}\right) \rightarrow S Y T\left(b^{a-1}(b-1)\right)$ by letting $d(U)$ be the (standard) tableau obtained by deleting the $n$ in the lower right hand corner of $U$.

Lemma 3.1 For any $U, T \in S Y T\left(b^{a}\right)$, we have that $\mu[U, T]=\mu[d(U), d(T)]$.

It is well known (see (6)) that $\mu[e(P), e(Q)]=\mu[P, Q]$ for any standard tableaux $P$ and $Q$ having the same arbitrary shape. We use this fact and the above result to get the desired fact about the action of $j$.

Proposition 3.2 Let $P, Q$ be standard tableaux which are either both in $S Y T\left(b^{a}\right)$ or $S Y T\left(b^{a-1}(b-1)\right)$. We have that $\mu[P, Q]=\mu[j(P), j(Q)]$.

This lemma says that, in the special case that our shape is a rectangle or a rectangle missing its outer corner, the action of promotion preserves the $\mu$ function. This does not hold in general for other shapes $(\lambda=(3,1)$ is a counterexample) .

To better understand the action of promotion, we introduce a new combinatorial set related to rectangular tableaux. Given a standard tableau $P$ of rectangular shape $b^{a}$, define the extended descent set of $P$ 
$D_{e}(P) \subseteq[n]$, as follows. For $1 \leq i \leq n-1$, say that $i \in D_{e}(P)$ if and only if $i$ is in the ordinary descent set $D(P)$. Let $U$ be the tableau with entries $\{2,3, \ldots, n\}$ obtained by deleting the 1 in $P$ and playing jeu-de-taquin to move the resulting hole to the lower right hand corner. The entry $n$ is either immediately above or immediately to the left of the hole in the lower right hand corner of $U$. Say that $n$ is contained in $D_{e}(P)$ if and only if $n$ appears above this hole in $U . D_{e}(P)$ is therefore the ordinary descent set $D(P) \subseteq[n-1]$, with the possible addition of the letter $n$.

Lemma 3.3 Let $P \in S Y T\left(b^{a}\right)$. For any $i, i \in D_{e}(P)(\bmod n)$ if and only if $i+1 \in D_{e}(j(P))(\bmod n)$.

We record a technical lemma about the image of the long cycle under the KL representation. Its proof is straightforward, albeit somewhat tedious. For the relevant definitions, see (17).

Lemma 3.4 Let $c_{n}=(1,2, \ldots, n) \in S_{n}$ be the long cycle. Let $\lambda=b^{a}$ be a rectangle. Identify permutations with their images under $R S K$. The coefficient of the KL basis element $C_{(j(C S S(\lambda)), C S S(\lambda))}^{\prime}(1)$ in the expansion of $c_{n} C_{(C S S(\lambda), C S S(\lambda))}^{\prime}(1)$ in the KL basis of $\mathbb{C}\left[S_{n}\right]$ is $(-1)^{a-1}$.

We now have all of the ingredients necessary to analyze the relationship between $j$ and the KL representation. Specifically, we show that up to a predictable scalar, $j$ acts like the long cycle $(1,2, \ldots, n) \in S_{n}$.

Proposition 3.5 Let $\lambda=b^{a}$ be a rectangular shape with $a b=n$ and let $\rho: S_{n} \rightarrow G L\left(S^{\lambda}\right)$ be the associated KL cellular representation, with basis identified with $S Y T(\lambda)$. Let $c_{n}$ denote the long cycle $(1,2, \ldots n) \in S_{n}$. Define a $\mathbb{C}$-linear map $J: S^{\lambda} \rightarrow S^{\lambda}$ by extending $J(P)=j(P)$.

We have that $\rho\left(c_{n}\right)=(-1)^{a-1} J$.

Proof: We show that for $i=1,2, \ldots, n-2$ we have

$$
J^{-1} \rho\left(c_{n}\right) \rho\left(s_{i}\right)=\rho\left(s_{i}\right) J^{-1} \rho\left(c_{n}\right) .
$$

Given a standard tableau $P$, let $j^{-1}(P)$ be the unique tableau which maps to $P$ under promotion. From the corresponding conjugation relation in the symmetric group it follows that

$$
\rho\left(c_{n}\right) \rho\left(s_{i}\right)=\rho\left(s_{i+1}\right) \rho\left(c_{n}\right) .
$$

On the other hand, because $1 \leq i \leq n-2$, we have that for any $P \in S Y T(\lambda)$,

$$
\begin{aligned}
J^{-1} \rho\left(s_{i+1}\right)(P) & = \begin{cases}-J^{-1}(P) & \text { if } i+1 \in D_{e}(P) \\
J^{-1}(P)+\sum_{i+1 \in D_{e}(Q)} \mu[P, Q] J^{-1}(Q) & \text { if } i+1 \notin D_{e}(P)\end{cases} \\
& = \begin{cases}-j^{-1}(P) & \text { if } i \in D_{e}\left(j^{-1}(P)\right) \\
j^{-1}(P)+\sum_{i \in D_{e}\left(j^{-1}(Q)\right)} \mu[P, Q] j^{-1}(Q) & \text { if } i \notin D_{e}\left(j^{-1}(P)\right)\end{cases} \\
& = \begin{cases}-j^{-1}(P) & \text { if } i \in D_{e}\left(j^{-1}(P)\right) \\
j^{-1}(P)+\sum_{i \in D_{e}\left(j^{-1}(Q)\right)} \mu\left[j^{-1}(P), j^{-1}(Q)\right] & \text { if } i \notin D_{e}\left(j^{-1}(P)\right)\end{cases} \\
& =\rho\left(s_{i}\right) J^{-1}(P) .
\end{aligned}
$$


The first equality is the definition of the KL representation, the second is Proposition 3.2, the third is Lemma 3.3, and the fourth is again the definition of the KL representation.

The above discussion implies that the operator $J^{-1} \rho\left(c_{n}\right)$ commutes with the action of the parabolic subgroup $S_{n-1}$ of $S_{n}$ on the irreducible $S_{n}$-module $S^{\lambda}$. Since $\lambda$ is a rectangle, $\lambda$ has a unique outer corner and by the branching rule for symmetric groups, the restriction $S^{\lambda} \downarrow_{S_{n-1}}^{S_{n}}$ remains an irreducible $S_{n-1}$-module. Therefore, by Schur's Lemma, we conclude that there is a number $\gamma \in \mathbb{C}$ so that

$$
J=\gamma \rho\left(c_{n}\right) .
$$

We want to show that $\gamma=(-1)^{a-1}$. This follows from Lemma 3.4.

The above result states that for Specht modules of rectangular shape the image of the long cycle $c_{n}$ under the KL representation is plus or minus the permutation matrix which encodes jeu-de-taquin promotion. It is not true that any conjugate of $c_{n}$ in $S_{n}$ enjoys this property - indeed, many are not even permutation matrices. Observe the analogy to Theorem 1.1 which states that the image of the long element $w_{o}$ under the KL representation for an arbitrary shape is plus or minus a permutation matrix which encodes evacuation.

Also notice that if $\lambda$ is a partition of any nonrectangular shape, then $\lambda$ has more than one outer corner. This implies that the restricted module $S^{\lambda} \downarrow_{S_{n-1}}^{S_{n}}$ is not irreducible and the above proof breaks down.

As a corollary to this we get a result due to Haiman (10) on the order of promotion.

Corollary 3.6 For $\lambda \vdash n$ rectangular, every element of $S Y T(\lambda)$ is fixed by $j^{n}$.

The order of promotion on $S Y T(\lambda)$ for arbitrary shapes $\lambda$ is unknown. Haiman proved the converse of the above result, as well.

Let $s_{n}$ denote the "affine" transposition $(1, n)$ in $S_{n}$. As another corollary, we get a formula for the image of $s_{n}$ under the KL representation in terms of the extended descent set.

Corollary 3.7 Let $\lambda=b^{a}$ be a rectangular shape and identify the basis of the corresponding left KL cellular representation with $S Y T(\lambda)$.

We have that

$$
s_{n} P= \begin{cases}-P & \text { if } n \in D_{e}(P) \\ P+\sum_{n \in D_{e}(Q)} \mu[P, Q] Q & \text { if } n \notin D_{e}(P) .\end{cases}
$$

Proof: Using the notation of the proof of the above proposition, we have that $s_{n-1}$ commutes with the action of $J^{-1} \rho\left(c_{n}\right)$. It follows that $J^{-1} s_{n}(P)=s_{n-1} J^{-1}(P)$. The desired formula follows.

Finally, the above analysis leads to many equalities of $\mu$ coefficients.

Corollary 3.8 Let $\lambda \vdash n$ be a rectangle and let $Y$ and $Y^{\prime}$ be two orbits of $S Y T(\lambda)$ under the action of $j$ which have relatively prime orders. Given $P, Q \in Y$ and $P^{\prime}, Q^{\prime} \in Y^{\prime}$ we have that $\mu\left[P, P^{\prime}\right]=\mu\left[Q, Q^{\prime}\right]$. 
For example, when $n=6$ and $\lambda=(2,2,2)$, we have that $|S Y T(\lambda)|=5$ and the cyclic action of $j$ on $S Y T(\lambda)$ breaks $S Y T(\lambda)$ into one cycle of size 2 and one cycle of size 3 . Explicitly, the operator $j$ acts on $\operatorname{SYT}((2,2,2))$ via:

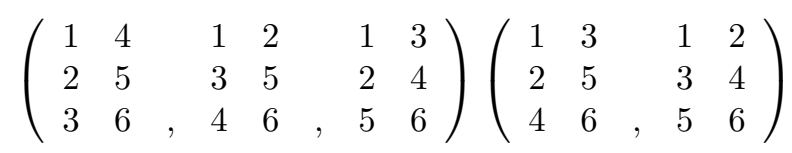

Mapping the elements of these orbits to their column reading words in $S_{6}$, the above Corollary implies that when $v$ is any element of the set $\{321654,521643,431652\} \subset S_{6}$ and $w$ is any element of the set $\{421653,531642\}$ we have that $\mu[v, w]$ is a constant. Since 421653 covers 321654 in Bruhat order, we see that this common value of $\mu$ is equal to 1 .

An application of the above proposition yields our desired result on cyclic sieving in the action of jeu-de-taquin on standard tableaux.

Theorem 3.9 Let $\lambda=b^{a}$ be a rectangular shape, $C=\mathbb{Z} / n \mathbb{Z}$ act on $X=S Y T(\lambda)$ by jeu-de-taquin promotion, and $X(q)=f^{\lambda}(q)$ be the $q$-hook length formula.

The triple $(X, C, X(q))$ exhibits the cyclic sieving phenomenon.

In the above example of the action of promotion on $\operatorname{SYT}((2,2,2))$, we have that

$$
f^{(2,2,2)}(q)=\frac{[6 !]_{q}}{[4]_{q}[3]_{q}[2]_{q}[3]_{q}[2]_{q}[1]_{q}}=\left(1-q+q^{2}\right)\left(1+q+q^{2}+q^{3}+q^{4}\right) .
$$

Letting $\zeta=e^{\frac{\pi i}{3}}$, we compute directly that

$$
\begin{array}{ccc}
f^{(2,2,2)}(1)=5 & f^{(2,2,2)}(\zeta)=0 & f^{(2,2,2)}\left(\zeta^{2}\right)=2 \\
f^{(2,2,2)}\left(\zeta^{3}\right)=3 & f^{(2,2,2)}\left(\zeta^{4}\right)=2 & f^{(2,2,2)}\left(\zeta^{5}\right)=0 .
\end{array}
$$

This is in agreement with the fixed point set sizes:

$$
\begin{array}{lll}
\left|S Y T((2,2,2))^{1}\right|=5 & \left|S Y T((2,2,2))^{j}\right|=0 & \left|S Y T((2,2,2))^{j^{2}}\right|=2 \\
\left|S Y T((2,2,2))^{j^{3}}\right|=3 & \left|S Y T((2,2,2))^{j^{4}}\right|=2 & \left|S Y T((2,2,2))^{j^{5}}\right|=0
\end{array}
$$

as predicted by Theorem 3.9 .

Proof: (Sketch) By Corollary 3.6, C does indeed act on X by promotion. Let $\zeta=e^{\frac{2 \pi i}{n}}$. Viewing $c=$ $(1,2, \ldots, n)$ as a permutation matrix in $S_{n} \subset G L_{n}(\mathbb{C})$, we get that $c$ is conjugate to $\operatorname{diag}\left(1, \zeta, \zeta^{2}, \ldots, \zeta^{n-1}\right)$. This means that for any $d \geq 0, c^{d}$ is conjugate to $\operatorname{diag}\left(1, \zeta^{d}, \zeta^{2 d}, \ldots, \zeta^{d(n-1)}\right)$.

Let $\chi^{\lambda}$ denote the character of the irreducible representation of $S_{n}$ corresponding of $\lambda$ A result of Springer (21) on the fake degree polynomial for the coinvariant algebra of type $A_{n-1}$ implies that, for any $d \geq 0$, we have the formula $\chi^{\lambda}\left(c^{d}\right)=\zeta^{d \kappa(\lambda)} f^{\lambda}\left(\zeta^{d}\right)$, where $\kappa(\lambda)=0 \lambda_{1}+1 \lambda_{2}+2 \lambda_{3}+\cdots$. Since $\lambda=b^{a}$, we can compute that $\kappa(\lambda)=\frac{b a(a-1)}{2}$, which implies that $\zeta^{d \kappa(\lambda)}=(-1)^{d(a-1)}$.

On the other hand by Proposition 3.5, $\chi^{\lambda}\left(c^{d}\right)$ is equal to the trace of $(-1)^{d(a-1)} J^{d}$, where $J$ is the permutation matrix which records promotion. This trace is $(-1)^{d(a-1)}$ times the number of fixed points $\left|X^{j^{d}}\right|$ of the action of $j^{d}$ on $X$. The desired CSP follows. 


\section{Promotion on Column Strict Tableaux}

The goal in this section is to extend the results of the last section concerning rectangular standard tableaux to the more general setting of rectangular column strict tableaux. To do this, we will use Skandera's (20) characterization of the dual canonical basis of $\mathbb{C}\left[x_{11}, \ldots, x_{n n}\right]$. In order to present this characterization, we introduce the notion of immanants.

For a positive integer $n \in \mathbb{N}$, let $x=\left(x_{i j}\right)_{1 \leq i, j \leq n}$ be an $n \times n$ matrix of commuting variables and let $\mathbb{C}\left[x_{11}, \ldots, x_{n n}\right]$ be the complex polynomial ring in these variables. We will sometimes abbreviate the latter ring as $\mathbb{C}\left[x_{i j}\right]_{1 \leq i, j \leq n}$. Call a polynomial in $\mathbb{C}\left[x_{i j}\right]_{1 \leq i, j \leq n}$ an immanant if it belongs to the $\mathbb{C}$-linear span of the permutation monomials $\left\{x_{1, w(1)} \cdots x_{n, w(n)} \mid w \in S_{n}\right\}$. Thus, immanants form an $n$ !-dimensional complex vector space. Given any polynomial $f\left(x_{11}, \ldots, x_{n n}\right) \in \mathbb{C}\left[x_{i j}\right]_{1 \leq i, j \leq n}$ and an $n \times n$ matrix $A=\left(a_{i j}\right)$ with entries in any commutative $\mathbb{C}$-algebra $R$, define $f(A)$ to be the element $f\left(a_{11}, \ldots, a_{n n}\right)$ of $R$ obtained by applying $f$ to $A$.

Following (18), define for any $w \in S_{n}$ the $w$-Kazhdan-Lusztig immanant $\operatorname{Imm}_{w}(x)$ by the equation

$$
\operatorname{Imm}_{w}(x)=\sum_{v \geq w}(-1)^{\ell(w, v)} P_{w_{o} v, w_{o} w}(1) x_{1, v(1)} \cdots x_{n, v(n)} .
$$

Specializing to the identity permutation, we have that $\operatorname{Imm}_{1}(x)=\operatorname{det}(x)$.

More generally, given any pair of compositions $\alpha, \beta \models n$ with, we define the matrix $x_{\alpha, \beta}$ to be $\left(x_{\alpha(i), \beta(j)}\right)_{1 \leq i, j \leq n}$. Note that either or both of $\ell(\alpha)$ or $\ell(\beta)$ may exceed $n$. We also construct the corresponding polynomial ring $\mathbb{C}\left[x_{\alpha(i), \beta(j)}\right]_{1 \leq i, j \leq n}$. For a permutation $w \in S_{n}$, denote by $\operatorname{Imm}_{w}\left(x_{\alpha, \beta}\right)$ the element of $\mathbb{C}\left[x_{\alpha(i), \beta(j)}\right]_{1 \leq i, j \leq n}$ which results in applying the $w$-Kazhdan-Lusztig immanant to the matrix $x_{\alpha, \beta}$. So, for example, we have that $\operatorname{Imm}_{w}(x)=\operatorname{Imm}_{w}\left(x_{1^{n}, 1^{n}}\right)$. In this paper we will mostly be interested in the case where $\beta=1^{n}$. Skandera showed that Lusztig's dual canonical basis can be constructed from polynomials of the form $\operatorname{Imm}_{w}\left(x_{\alpha, \beta}\right)$, and we will use these polynomials in our results, as well.

Theorem 4.1 (Skandera (20)) Let $k \geq 0$. The nonzero elements of the set $\left\{\operatorname{Imm}_{w}\left(x_{\alpha, \beta}\right)\right\}$, where $w$ ranges over $S_{n}$ and $\alpha$ and $\beta$ range over all possible compositions of $n$ having length $k$, are linearly independent and a subset of the dual canonical basis of the polynomial ring $\mathbb{C}\left[x_{11}, \ldots x_{k k}\right]$ in $k^{2}$ variables.

Let $Y=\mathbb{C}^{k}$ and $Z=\mathbb{C}^{n}$ be two complex vector spaces of dimensions $k$ and $n$, respectively. Let $Y^{*}$ and $Z^{*}$ denote their dual spaces with standard bases $\left\{y_{1}, \ldots, y_{k}\right\}$ and $\left\{z_{1}, \ldots, z_{n}\right\}$, respectively. Now the tensor product $Y^{*} \otimes Z^{*}$ has basis $x_{i j}:=y_{i} \otimes z_{j}$, for $1 \leq i \leq k$ and $1 \leq j \leq n$. In this way, we identify the symmetric algebra $\operatorname{Sym}\left(Y^{*} \otimes Z^{*}\right)$ with the polynomial ring $\mathbb{C}\left[x_{i j}\right]_{1 \leq i \leq k, 1 \leq j \leq n}$. This space carries an action of the general linear group $G L(Y)=G L\left(\mathbb{C}^{k}\right)$, where matrices act on the first component of simple tensors by $g \cdot(f \otimes h):=\left(f g^{-1}\right) \otimes h$. Taking $n=k$ and $i \in[n-1]$, viewing the adjacent transposition $s_{i}$ as an element of $S_{n} \subset G L\left(\mathbb{C}^{n}\right)$, we quote a result from (18) about this action.

Lemma 4.2 Let $w \in S_{n}$. We have that

$$
s_{i} \operatorname{Imm}_{w}(x)= \begin{cases}-\operatorname{Imm}_{w}(x) & s_{i} w>w \\ \operatorname{Imm}_{w}(x)+\operatorname{Imm}_{s_{i} w}(x)+\sum_{s_{i} z>z} \mu(w, z) \operatorname{Imm}_{z}(x) & s_{i} w<w .\end{cases}
$$


One can show that the Kazhdan-Lusztig immanants form a basis for the vector space of immanants. In fact, identifying permutations with their images under RSK, for any $\lambda \vdash n$ and $T \in S Y T(\lambda)$, we have that the space

$$
W_{T, n, 1^{n}}:=\mathbb{C}\left\{\operatorname{Imm}_{(T, P)}(x) \mid P \in S Y T(\lambda)\right\} \oplus \bigoplus_{\nu>\text { dom } \lambda} \mathbb{C}\left\{\operatorname{Imm}_{(U, S)}(x) \mid U, S \in S Y T(\nu)\right\}
$$

is closed under the left action of $S_{n}$. Moreover, the quotient space

$$
V_{T, n, 1^{n}}:=W_{T, n, 1^{n}} /\left(\bigoplus_{\nu>\operatorname{dom} \lambda} \mathbb{C}\left\{\operatorname{Imm}_{(U, S)}(x) \mid U, S \in S Y T(\nu)\right\}\right)
$$

carries the irreducible $S_{n}$-representation corresponding to the shape $\lambda$. A basis for $V_{T, n, 1^{n}}$ is given by the image of the set $\left\{\operatorname{Imm}_{(T, P)}(x) \mid P \in S Y T(\lambda)\right\}$ under the canonical projection map. Letting $I_{1^{n}}(P)$ denote the image of $\operatorname{Imm}_{(T, P)}(x)$ under this projection, we can write this basis as $\left\{I_{1^{n}}(P) \mid P \in S Y T(\lambda)\right\}$. By a change of label argument, the representation of $S_{n}$ on the quotient space $V_{T, n, 1^{n}}$ does not depend on the choice of the tableau $T$, and is given by

$$
s_{i} I_{1^{n}}(P)= \begin{cases}-I_{1^{n}}(P) & \text { if } i \in D(P) \\ I_{1^{n}}(P)+\sum_{i \in D(Q)} \mu[P, Q] I_{1^{n}}(Q) & \text { if } 1 \notin D(P) .\end{cases}
$$

The next lemma computes the effect of multiplication by the long cycle in $S_{n}$ on $I_{1^{n}}(P)$ for rectangular shapes.

Lemma 4.3 Let $\lambda=b^{a}$ be a rectangle and let $P \in S Y T(\lambda)$. Let $c_{n}=(1,2, \ldots n) \in S_{n}$ be the long cycle. We have that $c_{n} I(P)=(-1)^{b-1} I_{1^{n}}(j(P))$.

Working towards our goal of understanding the action of promotion on semistandard tableaux, we define the operations of standardization and $\alpha$-semistandardization on tableaux. These operations give us a means to transform row strict tableaux into standard tableaux and vice versa, when possible.

It is always possible to transform a row strict tableau into a standard tableau. Given a partition $\lambda \vdash n$ and a row strict tableau $P \in R S T(\lambda, k, \alpha)$ for some $\alpha \models n$, define the standardization std $(P)$ of $P$ to be the element of $S Y T(\lambda)$ given by replacing the $\alpha_{1} 1 s$ in $P$ with the numbers $\left[1, \alpha_{1}\right]$ increasing down columns, replacing the $\alpha_{2} 2 s$ in $P$ with the numbers $\left[\alpha_{1}+1, \alpha_{1}+\alpha_{2}\right]$ increasing down columns, and so on.

Given $\lambda \vdash n$ and a standard tableau $T \in S Y T(\lambda)$ along with a composition $\alpha \models n$, say that $T$ is $\alpha$-semistandardizable if $D(T)$ contains the union of the intervals $\left[1, \alpha_{1}\right),\left[\alpha_{1}+1, \alpha_{2}\right), \ldots$ Define the $\alpha$-semistandardization rst $\alpha$ ( $T)$ of $T \alpha$-semistandardizable to be the element of $\operatorname{RST}(\lambda, k, \alpha)$ formed by replacing the numbers in $\left[1, \alpha_{1}\right]$ in $T$ by $1 s$, the numbers in $\left[\alpha_{1}+1, \alpha_{1}+\alpha_{2}\right]$ in $T$ by $2 s$, and so on.

Lemma 4.4 Let $T \in S Y T(\lambda)$ be $\alpha$-semistandardizable. We have that std $\left(\right.$ rst $\left._{\alpha}(T)\right)=T$. Moreover, if $U \in R S T(\lambda, k, \alpha)$, then $\operatorname{std}(U)$ is $\alpha$-standardizable.

Therefore, for any composition $\alpha \models n$, standardization injects $R S T(\lambda, k, \alpha)$ into $S Y T(\lambda)$ and $\alpha$ semistandardization gives a bijection between the $\alpha$-semistandardizable elements of $S Y T(\lambda)$ and $\operatorname{RST}(\lambda, k, \alpha)$. These operations relate to promotion in the following way. 
Lemma 4.5 Let $\lambda \vdash n$ be a rectangle and $\alpha=\left(\alpha_{1}, \ldots, \alpha_{k}\right) \models n$. We have the following equality of operators on $\operatorname{SYT}(\lambda)$ :

$$
j \circ r s t_{\alpha}=r s t_{c_{k} \dot{\alpha}} \circ j^{\alpha_{k}},
$$

where the right hand side is defined if and only if the left hand side is defined.

$\mathrm{KL}$ immanants are related to the above operations in the following way.

Lemma 4.6 Let $U, T \in S Y T(\lambda)$ and let $k \in \mathbb{N}$. We have that $\operatorname{Imm}_{(U, T)}\left(x_{\alpha, 1^{n}}\right)=0$ if and only if $U$ is not $\alpha$-semistandardizable. Moreover, the set $\left\{\operatorname{Imm}_{\left(U^{\prime}, T^{\prime}\right)}\left(x_{\alpha^{\prime}, 1^{n}}\right)\right\}$ ranging over all possible $\alpha^{\prime} \models n$ with $\ell\left(\alpha^{\prime}\right) \leq k, U^{\prime}, T^{\prime} \in S Y T(\lambda)$, and $U^{\prime}$ that are $\alpha^{\prime}$-semistandardizable, is linearly independent.

For rectangular shapes, the polynomials $\operatorname{Imm}_{(U, T)}\left(x_{\alpha, 1^{n}}\right)$ for standard tableaux $U$ which are $\alpha$-semistandardizable will project onto elements of a basis of a certain quotient space which will carry an $S_{k}$ action under which the long element $(1,2, \ldots, k)$ will act as (essentially) jeu-de-taquin promotion. To further work toward this result, given $\alpha \models n$, we define an epimorphism $\mathbb{C}$-algebras $\pi_{\alpha}: \mathbb{C}\left[x_{i j}\right]_{1 \leq i, j \leq n} \rightarrow \mathbb{C}\left[x_{\alpha(i) j}\right]_{1 \leq i, j \leq n}$ by the formula $\pi_{\mu}\left(x_{i j}\right)=x_{\alpha(i) j}$.

Lemma 4.7 Let $U, T \in S Y T(\lambda)$. We have that $\pi_{\alpha}\left(\operatorname{Imm}_{(U, T)}(x)\right)$ is nonzero if and only if $U$ is $\alpha$ semistandardizable, in which case $\pi_{\alpha}\left(\operatorname{Imm}_{(U, T)}(x)\right)=\operatorname{Imm}_{(U, T)}\left(x_{\alpha, 1^{n}}\right)$.

One of the main advantages of the epimorphisms $\pi_{\alpha}$ is that they relate the action of $S_{n}$ on $\mathbb{C}\left[x_{i j}\right]_{1 \leq i, j \leq n}$ and the action of $S_{k}$ on $\mathbb{C}\left[x_{\alpha(i) j}\right]_{1 \leq i \leq k, 1 \leq j \leq n}$. This is made precise in the following lemma.

Lemma 4.8 Let $c_{n}=(1,2, \ldots, n)$ be the long cycle in $S_{n}$ and let $c_{k}=(1,2, \ldots, k)$ be the long cycle in $S_{k}$. For $\alpha=\left(\alpha_{1}, \ldots, \alpha_{k}\right) \models n$ we have a left action of $c_{n}$ on $\mathbb{C}\left[x_{i j}\right]_{1 \leq i, j \leq n}$ and $c_{k}$ maps $\mathbb{C}\left[x_{\alpha(i) j}\right]_{1 \leq i, j \leq n}$ into $\mathbb{C}\left[x_{c_{k} \dot{\alpha}(i) j}\right]_{1 \leq i, j \leq n}$. We have the following commutative square.

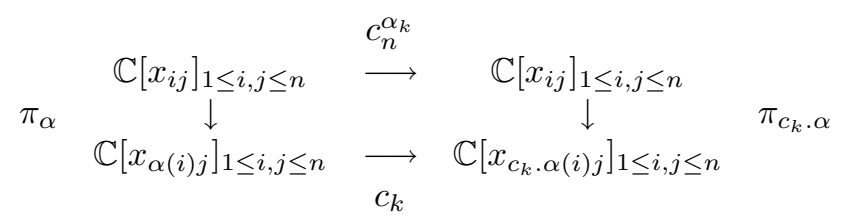

We are now in a position to define the $S_{k}$-modules which will yield our desired result. For any $T \in$ $S Y T(\lambda)$, define $W_{T, k}^{\prime}$ to be the space

$$
\mathbb{C}\left\{\operatorname{Imm}_{(T, U)}\left(x_{\alpha, 1^{n}}\right)\right\} \oplus \underset{\nu<<_{\text {dom }} \lambda}{ } \mathbb{C}\left\{\operatorname{Imm}_{(P, Q)}\left(x_{\alpha^{\prime}, 1^{n}}\right)\right\},
$$

where the first set ranges over all compositions $\alpha \models n$ such that $\ell(\alpha) \leq k$ and all $U \in S Y T(\lambda)$ which are $\alpha$-semistandardizable and the second set ranges over all compositions $\alpha^{\prime} \models n$ with $\ell\left(\alpha^{\prime}\right) \leq k$ and all pairs of tableaux $P, Q \in S Y T(\nu)$.

Lemma 4.9 $W_{T, k}^{\prime}$ is a left $G L\left(\mathbb{C}^{k}\right)$-module. 
We define $V_{T, k}$ to be the quotient $S_{k}$-module given by

$$
W_{T, k}^{\prime} / \bigoplus_{\nu>>_{d o m} \lambda} \mathbb{C}\left\{\operatorname{Imm}_{(P, Q)}\left(x_{\alpha^{\prime}, 1^{n}}\right)\right\}
$$

Basis elements of $V_{T, k}$ are given by the images of the polynomials $\operatorname{Imm}_{(U, T)}\left(x_{\alpha, 1^{n}}\right)$ for compositions $\alpha=\left(\alpha_{1}, \ldots, \alpha_{k}\right) \models n$ and $\alpha$-semistandardizable $U$. The image of the above polynomial in $V_{T, k}$ shall be abbreviated $I_{\alpha}\left(U^{\prime}\right)$, where $U^{\prime}$ is the unique element of $R S T(\lambda, k, \alpha)$ such that $\operatorname{std}\left(U^{\prime}\right)=U$. For $\alpha$ fixed, define the space $V_{T, k, \alpha}$ to be the span of all the $I_{\alpha}\left(U^{\prime}\right)$ for $\alpha$-semistandardizable $U$. The commutative square in Lemma 4.8 induces the following commutative square, where the maps induced by $\pi_{\alpha}$ are also called $\pi_{\alpha}$.

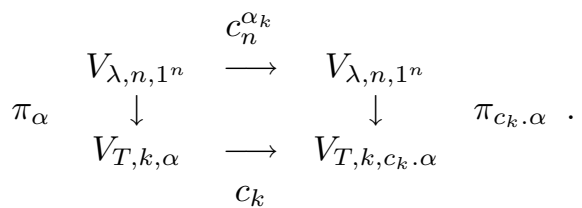

Using this square, we can relate the action of $c_{k}$ on $V_{T, k}$ to promotion.

Proposition 4.10 Given $U \in R S T(\lambda, k, \alpha)$ for $\lambda=b^{a}$ rectangular and $\alpha=\left(\alpha_{1}, \ldots, \alpha_{k}\right) \models n$, we have that $c_{k} I_{\alpha}(U)=(-1)^{\alpha_{k}(b-1)} I_{c_{k} \cdot \alpha}(j(U))$.

Corollary 4.11 For $\lambda$ rectangular, the order of $j$ on $\operatorname{RST}(\lambda, k)($ or $C S T(\lambda, k))$ is equal to $k$ unless $\lambda$ consists of a single column and $k=|\lambda|$.

For example, if $\lambda=(2,2)$ and $k=3$, the set $\operatorname{CST}((2,2), 3)$ contains 6 elements and promotion acts as the following permutation:

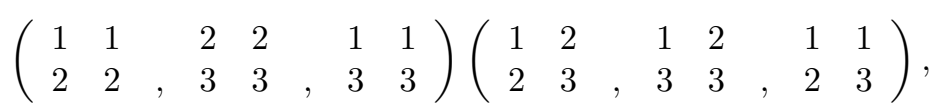

which does indeed have order $k=3$.

Theorem 4.12 Let $\lambda=b^{a}$ be a rectangular partition of $n, k \in \mathbb{N}$, and $X=C S T(\lambda, k)$. Let $C=\mathbb{Z} / k \mathbb{Z}$ act on $X$ by jeu-de-taquin promotion. Let $X(q)=q^{-\kappa(\lambda)} s_{\lambda}\left(1, q, q^{2}, \ldots, q^{k-1}\right)$.

The triple $(X, C, X(q))$ exhibits the cyclic sieving phenomenon.

The proof of this CSP uses the representations $V_{T, k}$ and is similar in spirit to the proof of Theorem 3.9. Keeping with the earlier example of $\lambda=(2,2)$ and $k=3$, we compute that $\kappa((2,2))=2$ and $s_{(2,2)}\left(x_{1}, x_{2}, x_{3}\right)=x_{1}^{2} x_{2}^{2}+x_{2}^{2} x_{3}^{2}+x_{1}^{2} x_{3}^{2}+x_{1} x_{2}^{2} x_{3}+x_{1} x_{2} x_{3}^{2}+x_{1}^{2} x_{2} x_{3}$. Maintaining the notation of Theorem 4.12, we therefore have that $X(q)=1+q+2 q^{2}+q^{3}+q^{4}$. Letting $\zeta=e^{\frac{2 \pi i}{3}}$, we see that

$$
X(1)=6 \quad X(\zeta)=0 \quad X\left(\zeta^{2}\right)=0 .
$$

These numbers agree with the fixed point set sizes:

$$
\left|C S T((2,2), 3)^{1}\right|=6 \quad\left|C S T((2,2), 3)^{j}\right|=0 \quad\left|C S T((2,2), 3)^{j^{2}}\right|=0,
$$


as predicted by Theorem 4.12 .

As a pair of corollaries, we get CSPs which were originally proven by Reiner, Stanton, and White in (15).

Corollary 4.13 Let $1 \leq k \leq n$ and let $X$ be the set of $k$-subsets of $[n]$. Let the cyclic group $C=\mathbb{Z} / n \mathbb{Z}$ act on $X$ by the long cycle $(1,2, \ldots, n) \in S_{n}$. Let $X(q)=\left[\begin{array}{l}n \\ k\end{array}\right]_{q}$ be the q-analogue of the binomial coefficient $\left(\begin{array}{l}n \\ k\end{array}\right)$.

Then, the triple $(X, C, X(q))$ exhibits the cyclic sieving phenomenon.

Corollary 4.14 Let $k$ and $n$ be positive integers and let $X$ be the set of all multisets of size $k$ with elements in $[n]$. Let the cyclic group $C=\mathbb{Z} / n \mathbb{Z}$ act on $X$ by the long cycle $(1,2, \ldots, n) \in S_{n}$. Let $X(q)=\left[\begin{array}{c}n+k \\ n\end{array}\right]_{q}$ be the q-analogue of the binomial coefficient $\left(\begin{array}{c}n+k \\ k\end{array}\right)$.

Then, the triple $(X, C, X(q))$ exhibits the cyclic sieving phenomenon.

Fix a rectangular partition $\lambda=b^{a}$ with $a b=n$, a positive integer $k$ and a composition $\alpha \models n$ with $\ell(\alpha)=k$ such that $\alpha$ has some cyclic symmetry. That is, there is an integer $d \mid k$ such that $\alpha_{i}=\alpha_{j}$ whenever $i \equiv j(\bmod d)$. We have a fixed point enumeration involving the action of certain powers of promotion on the set $C S T(\lambda, k, \alpha)$ and the Kostka-Foulkes polynomials. In representation theoretic terms, this corresponds to a weight space refinement of Theorem 4.12. Since $j$ maps the set $C S T(\lambda, k, \alpha)$ into the set $C S T\left(\lambda, k, c_{k} . \alpha\right)$, we have that the $d^{t h}$ power $j^{d}$ of $j$ maps the set $\operatorname{CST}(\lambda, k, \alpha)$ into itself. Note that for the special case $d=1, k=n$, and $\alpha=1^{n}$ this is the statement that $j$ acts on the set $S Y T(\lambda)$ of standard tableaux of shape $\lambda$. Since $j$ acts with order $k$ on the set $C S T(\lambda, k)$, we have that $j^{d}$ generates an action of the cyclic group $\mathbb{Z} /\left(\frac{k}{d} \mathbb{Z}\right)$ on $C S T(\lambda, k, \alpha)$.

For a partition $\lambda \vdash n$ and a composition $\alpha \models n$, let $K_{\lambda, \alpha}(q) \in \mathbb{N}[q]$ be the associated Kostka-Foulkes polynomial. The Kostka-Foulkes polynomials are $q$-analogues of the Kostka numbers $K_{\lambda, \alpha}$ which enumerate the number of column strict tableaux with shape $\lambda$ and content $\alpha$. In particular $K_{\lambda, \alpha}(1)=K_{\lambda, \alpha}$ always. The Kostka-Foulkes polynomials are the generating function for the charge statistic on tableaux and are also the coefficients of the change of basis matrix from Schur functions to Hall-Littlewood symmetric functions. For more details on these polynomials, see (14). The proof of this next theorem uses results on ribbon tableaux from (14).

Theorem 4.15 Let $\lambda=b^{a}$ be a rectangular partition of $n=a b$, $k$ be a positive integer, and $\alpha=$ $\left(\alpha_{1}, \ldots, \alpha_{k}\right)$ be a composition of $n$ of length $k$. Assume that there is some number $d \mid k$ so that $\alpha_{i}=\alpha_{j}$ whenever $i \equiv j(\bmod k)$. Let $\zeta \in \mathbb{C}$ be a root of unity of order $\frac{k}{d}$.

Then, for any $m \geq 0$, the number of fixed points $\left|C S T(\lambda, k, \alpha)^{j^{d^{m}}}\right|$ is equal to $\left|K_{\lambda, \alpha}\left(\zeta^{m}\right)\right|$.

\section{Dihedral Actions}

Let $\lambda=b^{a}$ be a rectangular partition with $a b=n$ and let $k \geq 0$. It is possible to show that we have eje $=j^{-1}$ as operators on $C S T(\lambda, k)$. Moreover, we know that the order of the operator $j$ is equal to $k$ while the order of the operator $e$ is equal to 2. This implies that the group $\langle e, j\rangle$ generated by $e$ and 
$j$, considered as a subgroup of the symmetric group $S_{C S T(\lambda, k)}$, is dihedral of order $2 k$. Similar remarks hold for the action of $e$ and $j$ on $R S T(\lambda, k)$ and $S Y T(\lambda)$.

We have already determined that the action of $j$ on the above sets of tableaux is modeled by the action of the long cycle of an appropriate symmetric group on an appropriate module. By Stembridge-BerensteinZelevinsky, the action of $e$ can be modeled by the action of the long element $w_{o}$. So, corresponding to the dihedral group acting on the sets $\operatorname{CST}(\lambda, k), \operatorname{RST}(\lambda, k)$, and $S Y T(\lambda)$, we get an associated dihedral group generated by the long cycle and $w_{o}$ sitting inside $S_{k}, S_{k}$, and $S_{n}$, respectively, equipped with an epimorphism onto our group of combinatorial operators. Together with our previous results, this gives us the number of fixed points of the operators $e$ and $e j$ on standard tableaux as a character evaluation.

Proposition 5.1 Let $\lambda=b^{a}$ be a rectangular partition of $n$, let $c_{n}$ be the long cycle in $S_{n}$, and let $\chi^{\lambda}: S_{n} \rightarrow \mathbb{C}$ be the irreducible character of $S_{n}$ corresponding to $\lambda$.

We have that

$$
\begin{aligned}
\left|S Y T(\lambda)^{e}\right| & =\left|\chi^{\lambda}\left(w_{o}\right)\right| \\
\left|S Y T(\lambda)^{e j}\right| & =\left|\chi^{\lambda}\left(w_{o} c_{n}\right)\right|
\end{aligned}
$$

Extending the Stembridge-Berenstein-Zelevinsky result on evacuation to the dual canonical basis and a statement about modules over the general linear group, we get the following statement about column strict tableaux. Details about its proof can be found in (17), but are omitted for this extended abstract.

Theorem 5.2 Let $\lambda=b^{a}$ be a rectangular partition of $n$ and let $k \geq 0$. Assume that at $k$ is odd. We have that

$$
\begin{aligned}
\left|C S T(\lambda, k)^{e}\right| & =\left|s_{\lambda}\left(1,-1,1, \ldots,(-1)^{k-1}\right)\right| \\
& =\left|\operatorname{CST}(\lambda, k)^{e j}\right| .
\end{aligned}
$$

On the other hand, if $k$ is even, we have that

$$
\begin{aligned}
\left|C S T(\lambda, k)^{e}\right| & =\left|s_{\lambda}\left(1,-1,1, \ldots,(-1)^{k-1}\right)\right| \\
\left|C S T(\lambda, k)^{e j}\right| & = \begin{cases}\left|s_{\lambda}\left(1,-1, \ldots,(-1)^{k-3},(-1)^{k-2},(-1)^{k-2}\right)\right| & \text { if a and } b \text { are both even or odd } \\
\left|s_{\lambda}\left(1,-1, \ldots,(-1)^{k-1}\right)\right| & \text { otherwise }\end{cases}
\end{aligned}
$$

Proof: Omitted. The claims about the operator $e$ are Theorem 3.1 of (24).

\section{Square Shapes and Type B, $2 \times n$ Shapes and Handshake Pat- terns and Noncrossing Partitions}

In this section we apply our results on tableaux to get CSPs for other combinatorial objects. For a positive integer $n$, let $B_{n}$ denote the Coxeter group of signed permutations of $[n]$ having order $2^{n} n$ !. It is possible to show that reduced words for the long element of $B_{n}$ are mapped to other reduced words to the long element under cyclic rotation. 
Theorem 6.1 Let $X$ be the set of reduced words for the long element in $B_{n}$. Let $C=\mathbb{Z} / n^{2} \mathbb{Z}$ act on $X$ by cyclically rotating words. Let $X(q)=f^{n^{n}}(q)$ be the $q-$ hook length formula. We have that the triple $(X, C, X(q))$ exhibits the cyclic sieving phenomenon.

Proof: (Sketch) By (10), there is a bijection between standard tableaux of shape $n \times n$ and reduced expressions for the long element of $B_{n}$ under which jeu-de-taquin promotion maps to cyclic rotation. The desired CSP follows.

$\square$ We now apply our

results for $2 \times n$ standard tableaux to prove CSPs for handshake patterns and noncrossing partitions. This gives a new proof of a result of White (25) which is alluded to in (4), as well as a new proof of results of Heitsch (11) which have biological applications related to RNA secondary structure.

Given $n \in \mathbb{N}$, a handshake pattern of size $2 n$ consists of a circle around which the points $1,2, \ldots, 2 n$ are written clockwise and a perfect matching on the set $[2 n]$ such that, when drawn on the circle, none of the arcs in this matching intersect. This can be thought of as a way in which the people labelled $1,2, \ldots, 2 n$ can all shake hands so that no one crosses arms. Let $H_{n}$ denote the set of all handshake patterns of size $2 n$.

For $n \in \mathbb{N}$, a noncrossing partition of $[n]$ is a set partition $\left(P_{1}\left|P_{2}\right| \ldots \mid P_{k}\right)$ of $[n]$ so that whenever there are integers $a, b, c, d, i$, and $j$ with $1 \leq a<b<c<d \leq n$ and $a, c \in P_{i}$ and $b, d \in P_{j}$, we must necessarily have that $i=j$. Drawing the numbers $1,2, \ldots, n$ clockwise around a circle, a partition 1 of $[n]$ is noncrossing if and only if when the blocks of $\pi$ are drawn on the circle, none of the regions intersect.

The set $N C(n)$ of all noncrossing partitions of $[n]$ is a lattice with respect to the partial order given by refinement. The lattice $N C(n)$ is complemented, and given a noncrossing partition $\pi$ of $[n]$, Kreweras complementation (see (13) for its definition) gives a way to produce a complement of $\pi$ in $N C(n)$.

Both of the sets $H_{n}$ and $N C(n)$ have cardinality given by the Catalan number $C_{n}=\frac{1}{n+1}\left(\begin{array}{c}2 n \\ n\end{array}\right)$. Moreover, these sets both carry an action of the cyclic group of order $2 n$ - in the case of $H_{n}$ given by the action of rotating the table clockwise by one position and in the case of $N C(n)$ given by Kreweras complementation. As a corollary of our tableaux results, we get results of White (25) and Heitsch (11).

Theorem 6.2 Let $n \in \mathbb{N}$ and let $C=\mathbb{Z} /(2 n \mathbb{Z})$. Let $X(q)$ be the $q$-Catalan number

$$
X(q)=\frac{1}{[n+1]_{q}}\left[\begin{array}{c}
2 n \\
n
\end{array}\right]_{q} .
$$

Let $X$ be either $H_{n}$ equipped with the $C$-action of rotation or $N C(n)$ equipped with the $C$-action of Kreweras complementation.

Then, the triple $(X, C, X(q))$ exhibits the cyclic sieving phenomenon.

We also have a result concerning dihedral actions and character evaluations.

Proposition 6.3 Let $X$ be either the set $H_{n}$ or the set $N C(n)$. $X$ carries an action of $D_{4 n}$, where $r$ and $s$ have the action described above in the case of $H_{n}$ and act by reflection about the line through 1 bisecting the circle and Kreweras complementation in the case of $N C(n)$. Let $w_{o}$ denote the long element of $S_{2 n}$ and let $c_{2 n}$ denote the long cycle $(1,2, \ldots, 2 n)$ in $S_{2 n}$. 
Then, the number of fixed points of the operators $r$ and $r s$ on $X$ are given by the formulae:

$$
\begin{aligned}
\left|X^{r}\right| & =\left|\chi^{(n, n)}\left(w_{o}\right)\right| \\
\left|X^{r s}\right| & =\left|\chi^{(n, n)}\left(w_{o} c_{2 n}\right)\right|
\end{aligned}
$$

\section{Aknowledgements}

The author is grateful to Andrew Berget, Serge Fomin, Victor Reiner, and John Stembridge for helpful conversations.

\section{References}

[1] O. Abuzzahab, M. Korson, M. Li, and S. Meyer. Cyclic and dihedral sieving for plane partitions. 2005. U. Minnesota REU Report.

[2] H. Barcelo, V. Reiner, and D. Stanton. Bimahonian distributions. 2007.

[3] A. Berenstein and A. Zelevinsky. Canonical bases for the quantum groups of type $A_{r}$ and piecewise linear combinatorics. Duke Math. J., 82:473-502, 1996.

[4] D. Bessis and V. Reiner. Cyclic sieving of noncrossing partitions for complex reflection groups. Preprint math.CO/0701792 on ArXiv, 2007.

[5] S. C. Billey and G. Warrington. Kazhdan-Lusztig polynomials for 321-hexagon avoiding permutations. J. Algebraic Combin., 13(2):111-136, 2001.

[6] A. Björner and F. Brenti. Combinatorics of Coxeter groups. Springer, 1995.

[7] Francesco Brenti. A combinatorial formula for Kazhdan-Lusztig polynomials. Invent. Math., 118(2):371-394, 1994.

[8] Francesco Brenti. Combinatorial expansions of Kazhdan-Lusztig polynomials. J. London Math. Soc., 55(2):448-472, 1997.

[9] A. M. Garsia and T. J. McLarnan. Relations between Young's natural and the Kazhdan-Lusztig representations of $S_{n}$. Adv. Math., 69:32-92, 1988.

[10] M. Haiman. Dual equivalence with applications, including a conjecture of Proctor. Discrete Math., 99:79-113, 1992.

[11] C. E. Heitsch. Kreweras complementation and orbits in Catalan lattices. Submitted, 2007.

[12] D. Kazhdan and G. Lusztig. Representations of Coxeter groups and Hecke algebras. Inv. Math., 53:165-184, 1979.

[13] G. Kreweras. Sur les partitions non croisées d'un cycle. Discrete Math., 1(4):333-350, 1972. 
[14] A. Lascoux, B. Leclerc, and J.-Y. Thibon. Green polynomials and Hall-Littlewood functions at roots of unity. Euorp. J. Combinatorics, 15:173-180, 1994.

[15] V. Reiner, D. Stanton, and D. White. The cyclic sieving phenomenon. J. Combin. Theory Ser. A, 108, 2004.

[16] Victor Reiner, 2007. Personal communication.

[17] B. Rhoades. Cyclic sieving and promotion. 2007. In progress. Available at http://www.math.umn.edu/rhoad030/research .

[18] B. Rhoades and M. Skandera. Kazhdan-Lusztig immanants and products of matrix minors. $J$. Algebra, 304(2):793-811, 2006.

[19] B. Sagan. The Symmetric Group. Springer, New York, 2001.

[20] M. Skandera. On the dual canonical and Kazhdan-Lusztig bases and 3412, 4231-avoiding permutations. 2006. Submitted.

[21] T. A. Springer. Regular elements of finite reflection groups. Invent. Math., 29:159-198, 1974.

[22] R. Stanley. Enumerative Combinatorics, volume 1. Cambridge University Press, Cambridge, 1997.

[23] R. Stanley. Enumerative Combinatorics, volume 2. Cambridge University Press, Cambridge, 1999.

[24] J. Stembridge. Canonical bases and selfevacuating tableaux. Duke Math. J., 82(3):585-606, 1996.

[25] Dennis White, 2007. Personal communication. 
